\title{
UNE TECHNIQUE NOUVELLE D'UTILISATION DES PHOTOGRAPHIES HÉMISPHÉRIQUES POUR LA MESURE DU CLIMAT LUMINEUX EN FORÊT
}

\author{
M. BECKER \\ Laboratoire de Recherches de la Chaire de Botanique forestière. \\ Centre national de Recherches forestières, I.N.R.A., \\ 54 - Nancy
}

\section{RÉSUMÉ}

Le climat lumineux est une des variables écologiques les plus importantes, mais aussi íune des plus difficiles à mesurer en forêt.

La technique que nous proposons fait appel aux photographies hémisphériques (obtenues avec un objectif super grand angle de $180^{\circ}$ ). L'appareil photographique est placé horizontalement lors de la prise de vue. La pellicule utilisée est à grand contraste, afin de n'obtenir que des noirs (trouées dans le feuillage) et des blancs (matière végétale ou relief du terrain).

Les négatıfs ainsi obtenus sont contretypés; on mesure le coefficient de transmission global du diapositif (fig. 2) pour avoir le couvert angulaire de la station étudiée.

Pour obtenir une valeur approchée du rayonnement énergétique moyen annuel, il est nécessaire de distinguer rayonnement solaire diffus $(\mathrm{R} f)$ et rayonnement solaire direct $(\mathrm{R} r)$. A cette fin, il est nécessaire de faire deux mesures séparées en superposant au diapositif à analyser une cale optique "rayonnement diffus 》 (fig. 5), puis une cale "rayonnement direct 》 (fig. 8), toutes deux du même format que le diapositif.

La cale $\mathrm{R} f$ est circulaire et sinusoïdale; elle corrige le coefficient de transmission du diapositif pour tenir compte de la hauteur des trouées du couvert forestier au-dessus de l'horizon. Du centre à sa périphérie, le coefficient de transmission de la cale $\mathbf{R} f$ varie selon la loi $\mathrm{T}=\cos t(t=$ distance au centre, en degrés).

La cale $\mathrm{R} r$ doit tenir compte de la portion du ciel balayée annuellement par le soleil dans la région considérée (voir fig. 8). Dans la partie correspondante, son coefficient de transmission varie selon une loi un peu plus complexe qui tient compte, d'une part de la hauteur des trouées au-dessus de l'horizon, d'autre part de la masse atmosphérique $m$ et de son coefficient d'absorption :

$$
\mathrm{T}=(1,341)^{1-{ }^{m}} \cdot \cos t
$$

Il reste, d'une part à étudier la précision des mesures ainsi obtenues, par comparaison avec celles de thermopiles intégratrices maintenues en station pendant le longues périodes, d'autre part à mettre au point une technique suffisamment simple de correction en fonction de la pente et de l'exposition du terrain. 


\section{I. - INTRODUCTION}

On sait que les études phyto-écologiques comportent toujours un grand nombre de points d'observations où sont recueillies de nombreuses données, floristiques ef écologiques.

En milieu forestier, une des variables écologiques parmi les plus importantes, mass aussi les plus problématiques, concerne le micro-climat lumineux des stations inventoriées (Aussenac, Becker, 1968 ; Becker, 1969 ; PicArd, 1970).

Les techniques d'interprétation des donnée; recueillies sur le terrain font appel à des méthodes statistiques qui ne trouvent leur pleine efficacité que dans le cas de variables quantitatives, réellement mesurées. Or, en ce qui concerne la lumière, nous devons actuellement nous contenter d'une simple appréciation, avec tout ce que cela comporte de subjectif, du «couvert angulaire», c'est-à-dire du pourcentage de ciel occulté par le relief et la végétation.

La technique exposée a pour but de remplacer cette appréciation par une réelle mesure, plus fine et qui élimine les risques de biais dus à l'opérateur. Compte tenu du grand nombre de stations inventoriées dans ce type d'étude, cette technique, comme toutes celles utilisées pour les autres variables, nécessite d'être relativement simple et en tout cas facile et rapide à mettre en œuvre sur le terrain, sans exiger de matériel lourd ou encombrant.

Rappelons d'autre part que, parmi les autres variables mesurées, figurent la pente et l'exposition, dont la mesure a pour but essentiel d'apporter un correctif en ce qui concerne le rayonnement solaire direct. La technique proposée devrait permettre ulterieurement de synthétiser en une seule valeur les données relatives au couvert, à la pente et à l'exposition.

\section{II. - LES PHOTOGRAPHIES HÉMISPHÉRIQUES}

Les photographies hémisphériques sont obtenues à l'aide d'un appareil muni d'un objectif super grand angle (jusqu'à $180^{\circ}$ ), dit « œil de poisson» ou «fish-eye»(1). L'idée de les utiliser pour étudier, depuis le sol, le couvert forestier est déjà ancienne (Evans, Coombe, 1959; Brown, 1962; Anderson, 1964 ; Brown, Worley, 1965 ; MAdGWick, BRumfield, 1969).

L'originalité de la technique proposée réside, d'une part dans le parti qu'on espère en tirer, d'autre part, et surtout, dans le processus d'interprétation des photographies obtenues. La plupart des auteurs ont cherché à estimer, à un instant donné, le rayonnement solaire direct et le rayonnement diffus pénétrant sous le couvert. Les photogra-

(1) Le boîtier photographique est un Nikon F. $(24 \times 36 \mathrm{~mm})$. L'objectif également de la marque Nikor ; ses caractéristiques techniques principales sont : ouverture $1: 1,5$; distance focale $f=$ $7,5 \mathrm{~mm}$. 
phies sont tirées sur papier; une des techniques (MADGwick, BrumfiEld) consiste à échantillonner convenablement la surface de la photographie et à mesurer, en chacun des points retenus, par réflexion, la densité optique. Les valeurs obtenues aux différents points peuvent être corrigées en fonstion de leur distance angulaire au zénith.

La technique qui va être décrite a pour but d'évaluer la quantité d'énergie parvenant au sol, en un point donné, sur l'ensemble d'une année moyenne. La photographie est analysée, non par réflexion, mais par transmission (sur un diapositif), et ceci de façon globale: deux mesures seulement sont nécessaires, l'une pour le rayonnement direct, l'autre pour le rayonnement diffus.

L'intérêt premier de cette démarche réside donc dans la rapidité des opérations de dépouillement, ce qui permet à cette mesure de prendre place parmi les autres mesures classiques du relevé phyto-écologique.

\section{III. - DESCRIPTION DE LA TECHNIQUE}

\section{1. - En forêt}

\subsection{L'appareillage photographique.}

Les images obtenues sur le négatif sont circulaires (ce qui est logique), de diamètre 23,2 mm. A l'aide d'un théodolite, nous avons vérifié expérimentalement que les distances des divers points de la photographie au centre de celle-ci sont, dans toutes les directions, rigoureusement proportionnelles aux angles correspondants dans l'espace photographié. Les angles solides sont donc restitués sans déformations dans l'ensemble du champ ; ceci est une propriété très importante, pour le parti qu'on espère tirer de cet objectif, qui peut être résumée par la formule simple (voir fig. 1) :

$$
1=k . t \text {. }
$$

$k$ est une constante propre à l'appareillage. Lorsque $t=90^{\circ}, 1=11,6 \mathrm{~mm}$ (rayon de la photographie sur le négatif obtenu), donc $k=0,129 \mathrm{~mm} /$ degré.

\subsection{Le film utilisé. Justifications.}

Pour répondre au but recherché, l'image obtenue d'un peuplement (que l'on supposera identique à lui-même d'un bout à l'autre de l'année (1)) doit être la même quelles que soient les conditions de lumière extérieure au peuplement, c'est-à-dire quelles que soient la saison, l'heure de la prise de vue et les conditions météorologiques du moment. Ceci répond à la notion de couvert angulaire précédemment évoquée ; il s'agit en fait de rechercher un contre-jour aussi parfait que possible, seules les taches de ciel présentes dans le couvert venant impressionner la pellicule.

(1) Dans la réalité, la morphologie de tout couvert évolue au long d'une année, surtout s'il s'agit de peuplements feuillus. Nous reviendrons sur ce point dans le $\$ 4$. 
On a donc intérêt à utiliser une pellicule à très fort contraste, du type de celles qui sont ordinairement employées pour la reproduction de dessins au trait. La pellicule que nous utilisons est du Microfile Kodak (sensibilité très faible, environ 6 ASA).

\subsection{La prise de vue. Précautions à prendre.}

Il s'est avéré indispensable de n'opérer qu'en l'absence d'ensoleillement direct sinon les troncs et les branches visibles frappées par le soleil d'une part - par réflexion -, certaines feuilles d'autre part — par transmission - , risquent d'être aussi lumineux, et même plus, que le ciel et d'impressionner la pellicule au même titre que les trouées dans le feuillage. Enfin, il peut apparaître un halo gênant à l'emplacement du soleil lui-même.

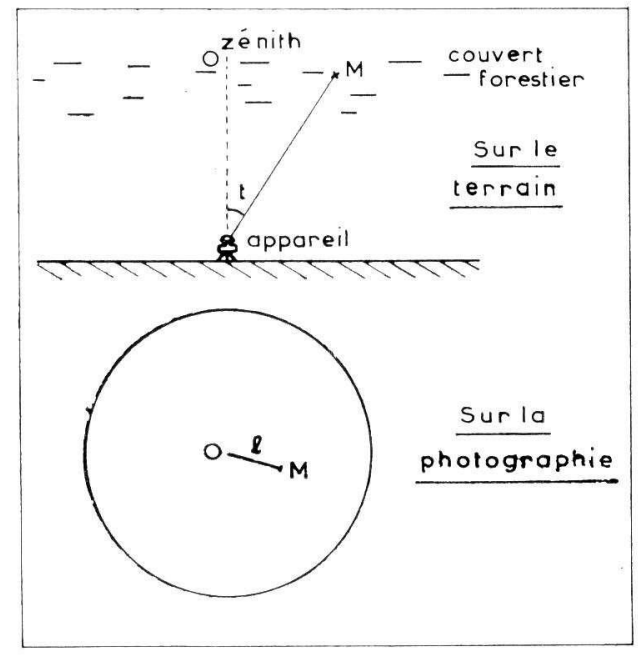

FIG. 1. - Restitution de la photographie hémisphérique : $l=k . t$

FIG. 1. - Restitution of the hemispherical photograph: $l=k . t$

Les conditions optimales de travail sont réunies par temps uniformément couvert, mais sans pluie.

Un des points les plus délicats à résoudre fut celui du réglage de l'appareil photographique, qui dépend de l'éclairement au-dessus du peuplement. On dispose en effet rarement d'une trouée suffisamment vaste et proche pour faire une mesure de référence avec une cellule photo-électrique classique. En fin de compte, la ditficulté a pu être aisément tournée grâce à un type de cellules photo-électriques existant depuis peu sur le marché ; il s'agit de cellules directionnelles, à visée reflex et angle de visée très faibio (un degré) (exemple : «Spot sensor» Soligor).

Pratiquement, il suffit alors de repérer la portion du ciel la moins lumineuse et de viser, avec cette cellule spéciale, l'intérieur d'une ou plusieurs petites trouées pour déterminer vitesse et nombre d'ouvertures à adopter. Il n'y a pas de réglage de distance. 
L'appareil est mis en station sur un pied photographique (1) ; un niveau à bulle permet de viser parfaitement le zénith. Il est nécessaire de convenir d'une position précise de l'appareil par rapport à la direction du nord magnétique, pour pouvoir ultérieurement orienter les photographies obtenues.

La figure 2 montre un exemple de photographie ainsi obtenue.

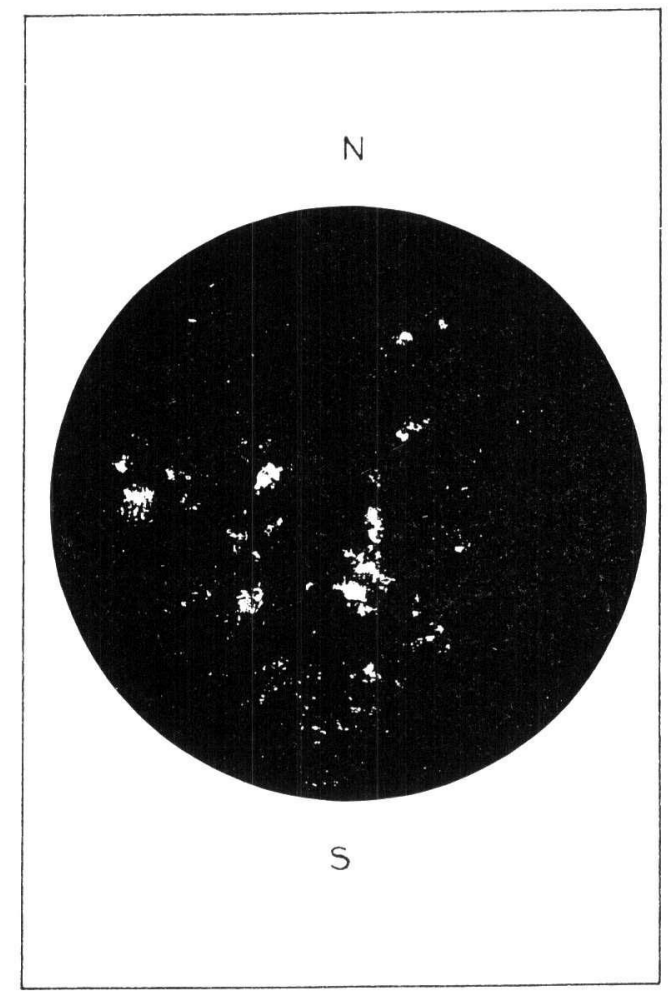

FIG. 2. - Un exemple de photographie hémisphérique (Taillis vieilli de Hêtre)

FIG. 2. - An exemple of hemispherical photograph (Old Beech coppice)

\section{2. - Analyse des photographies}

\subsection{Mesure du couvert angulaire seulement.}

Le film pris sur le terrain est développé à l'aide d'un révélateur à grand contraste (type Kodak D 11) afin d'accentuer l'effet de contre-jour, de «tout ou rien». Il est ensuite nécessaire de contretyper les négatifs obtenus, afin d'obtenir des diapositifs sus

(1) A la hauteur de la végétation que l'on désire étudier plus particulièrement; en général la strate herbacée $(0,50 \mathrm{~m}$ environ). 
lesquels le ciel apparaît transparent et la végétation opaque. Toujours dans le but évoqué ci-dessus, l'opération se fait avec le même type de pellicule et le même révélateur Les meilleurs résultats ne sont pas obtenus à l'aide d'un tirage par contact, mais par l'intermédiaire d'un appareil photographique, avec un rapport objet-image de $1 / 1$.

La mesure se fait à l'aide d'un lux-mètre, dont l'élément photo-sensible doit être de surface au moins égale à celle de la photographie (diamètre 23,2 mm), et d'une source de lumière délivrant un faisceau aussi homogène que possible.

Soit $x_{0}$ l'indication du lux-mètre lorsqu'on interpose un diapositif entièrement transparent tel qu'on l'obtiendrait par photographie en plein dézouvert) et $x$ si le diapositif correspond à une station sous couvert forestier. Le couvert angulaire c.a. en ce point (en \%) est :

$$
\text { c.a. }=\frac{x}{x_{0}} \cdot 100 .
$$

\subsection{Mesure des rayonnements direct et diffus.}

\subsection{Considérations générales.}

Nous admettrons avec Roussel (1962) que, au moins dans la moitié nord de la France, le rayonnement solaire direct et le rayonnement diffus interviennent chacun pour moitié environ dans le rayonnement total reçu au cours d'une année moyenne (1). Or, sous ces latitudes, un centimètre carré de sol horizontal reçoit environ 100.000 calories par an. Dans ce qui suit nous supposerons done qu'un $\mathrm{cm}^{2}$ de sol en station horizontale et découverte reçoit, au cours d'une année moyenne, 50.000 cal sous forme de rayonnement direct et $50.000 \mathrm{cal}$ sous forme de rayonnement diffus.

Ces considérations ne constituent pas cependant une limite à la méthode exposée ici. Toutes autres valeurs peuvent être utilisées, soit que celles citées dans notre texte s'avèrent imprécises, soit que l'on opère dans des contrées climatiquement différentes.

Sur le plan théorique, les techniques qui vont être proposées dans les deux paragraphes suivants découlent des études de Roussel (1952) concernant le climat lumineux de la trouée circulaire et de la bande en forêt. L'utilisation de la photographie hémisphérique permet, grâce à un artifice que nous allons exposer, d'appliquer les conclusions de l'auteur à des couverts de morphologie quelconque.

\subsection{Mesure du rayonnement diffus $R f$.}

La lumière diffuse provient de l'ensemble du ciel, nuageux ou dégagé, que l'on peut assimiler, en un point d'observation donné $P$ (fig. 3), à une demi-sphère de rayon unité.

Naturellement, la luminance du ciel, à un instant donné, n'est pas la même dans toutes les directions. Cependant, mise à part, semble-t-il, une baisse générale un peu marquée au Nord, on peut admettre qu'elle est statistiquement égale partout si l'on considère globalement l'ensemble d'une longue période, par exemple une année moyenne.

(1) Cette répartition simple, résulte, notamment, des travaux de BLACKWELL (1954) à Londres, et de Steinhauser, Eckel et Sauberer (1955) à Vienne. 
Soit une trouée dans le feuillage de surface élémentaire $d \mathrm{~S}$, située à un angle $t$ par rapport au zénith (fig. 3). L'éclairement en P est:

$$
d \mathrm{E}=\frac{\mathrm{L} \cdot d \mathrm{~S} \cdot \operatorname{cost} t}{r^{2}}=\mathrm{L} \cdot \cos t \cdot d \mathrm{~S} \text {. }
$$

L : luminance du ciel.

Pour l'ensemble des trouées situées au même angle $t$, on aura :

$$
\mathrm{E}_{t}=\text { L. S. } \cos t \text {. }
$$

Si une photographie hémisphérique est prise au point $P$, nous savons que $S$ sera représentée sur le négatif par une surface proportionnelle $s$ indépendante de $t$ (cf. $\S 3.11$ ).

$$
\mathrm{S}=\text { k.s } \quad \text { d'où } \quad \mathrm{E}_{t}=\text { L.k.s. } \cos t=\mathrm{K} . s \cdot \cos t .
$$

Il apparaît donc nécessaire, pour chaque valeur de $t$, de multiplier par cos $t$ le coefficient de transmission du négatif, ce qui semblerait interdire tout espoir de lecture globale de ce négatif.

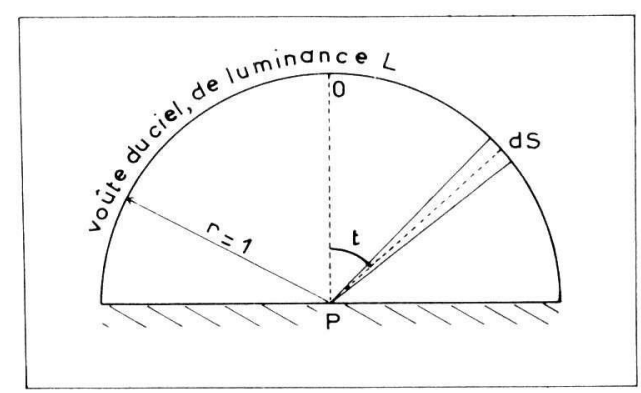

FIG. 3. - Calcul de l'éclairement en un point $P, \mathrm{~d} E=L . \cos \mathrm{t} . \mathrm{d} S$

FIG. 3. - Calculation of light at $P$ point : $\mathrm{d} E=L \cdot \cos \mathrm{t} . \mathrm{d} S$

La difficulté peut être tournée en superposant au négatif hémisphérique une cale optique circulaire de même diamètre, de coefficient de transmission variable $\mathrm{T}=\cos t$, $t$ variant de $0^{\circ}$ au centre de la cale (correspondant au zénith du négatif), à $90^{\circ}$ sur la périphérie (horizon du négatif).

Construction d'une cale circulaire sinusoïdale. - Il n'a pas été trouvé de procédé satisfaisant permettant de construire une cale répondant à cette loi de façon continue. Par contre, il est aisé de construire une cale discontinue en photographiant un négatif obtenu par superposition de cercles de rayons convenables, découpés dans des feuilles translucides de coefficient de transmission To connu et constant.

Le négatif de la cale, étant construit en grand format et appelé à être photographié pour obtenir la cale définitive de diamètre $23,2 \mathrm{~mm}$, doit être caractérisé par un coefficient de transmission qui suive la loi de variation $(1-\cos t)$.

Les coefficients de transmission des divers anneaux qui le forment sont, de l'extérieur vers l'intérieur:

$$
\text { 1, To, } \mathrm{To}^{2}, \mathrm{To}^{3}, \ldots, \mathrm{To}^{n}, \ldots
$$


Ces valeurs ne sont rigoureusement correctes que pour la partie médiane de chaque anneau. Le bord extérieur de chaque anneau, où se produit la discontinuité, correspond (de façon approchée) à la valeur théorique :

$$
\frac{\mathrm{To}^{n}+\mathrm{To}^{n-1}}{2}
$$

Or, cos $t=1-\mathrm{T}$; les rayons $t_{n}$ des divers cercles empilés les uns sur les autres sont donc tels que :

$$
\cos t_{n}=1-\frac{\mathrm{To}^{n}+\mathrm{To}^{n-1}}{2}
$$

Le négatif de la cale ainsi construite est photographié avec une pellicule restituant aussi bien que possible les diverses nuances de gris (type Plus X Kodak). Il est nécessaire de faire plusieurs clichés à des expositions différentes, de façon à choisir le cliché le mieux adapté. Ce choix est aisé et repose sur les considérations suivantes :

- supposons un cliché circulaire, de rayon $\frac{\pi}{2}$, entièrement et parfaitement transparent; si on projette dessus un faisceau de lumière homogène, la quantité de lumière Qo qu'il laissera passer sera proportionnelle à sa surface :

$$
\mathrm{Qo}=\mathrm{K} \cdot \pi \mathrm{R}^{2}=\mathrm{K} \cdot \frac{\pi^{3}}{4}
$$

- supposons la cale que nous voulons obtenir (fig. 4) placée dans les mêmes conditions ; une surface annulaire élémentaire de largeur $d t$, située à la distance $t$ du centre, laissera passer la quantité de lumière :

$$
d \mathrm{Q}=\mathrm{K} \cdot \cos t \cdot 2 \pi \cdot t \cdot d t .
$$

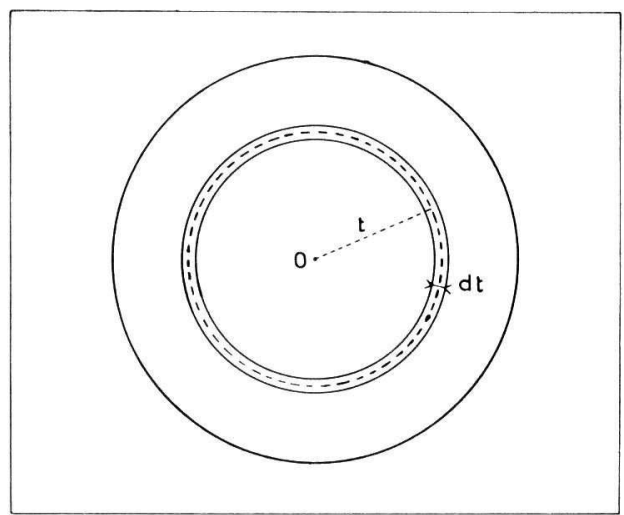

FIG. 4. - Calcul de la quantité de lumière que laisse passer un anneau de la cale optique $R \mathrm{f}: \mathrm{d} Q=2 \pi . K$. cos t.dt

FIG. 4. - Calculation of the light quantity passing through a ring of the Rf optical wedge : $\mathrm{d} Q=2 \pi . K$. cos t.dt 
Pour l'ensemble de la cale sinusoïdale :

$\mathrm{Q}=\int_{0}^{\frac{\pi}{2}} \mathrm{~K} \cdot \cos t \cdot 2 \pi \cdot t \cdot d t=2 \pi \cdot \mathrm{K} \cdot \int_{0}^{\frac{\pi}{2}} t \cdot \cos t \cdot d t$.
$\mathrm{Q}=2 \pi \mathrm{K}|t \sin t+\cos t| \frac{\pi}{2}=2 \pi \cdot \mathrm{K}\left(\frac{\pi}{2}-1\right)=\mathrm{K}\left(\pi^{2}-2 \pi\right)$.

Le rapport des coefficients de transmission globaux de ces deux cales doit donc être égal à :

$$
\frac{\mathrm{Q}}{\mathrm{Qo}}=\frac{4\left(\pi^{2}-2 \pi\right)}{\pi^{3}}=\frac{4(\pi-2)}{\pi^{2}} \cong 0,463 .
$$

La photographie de la figure 5 représente la cale circulaire sinusoïdale ainsi obtenue.

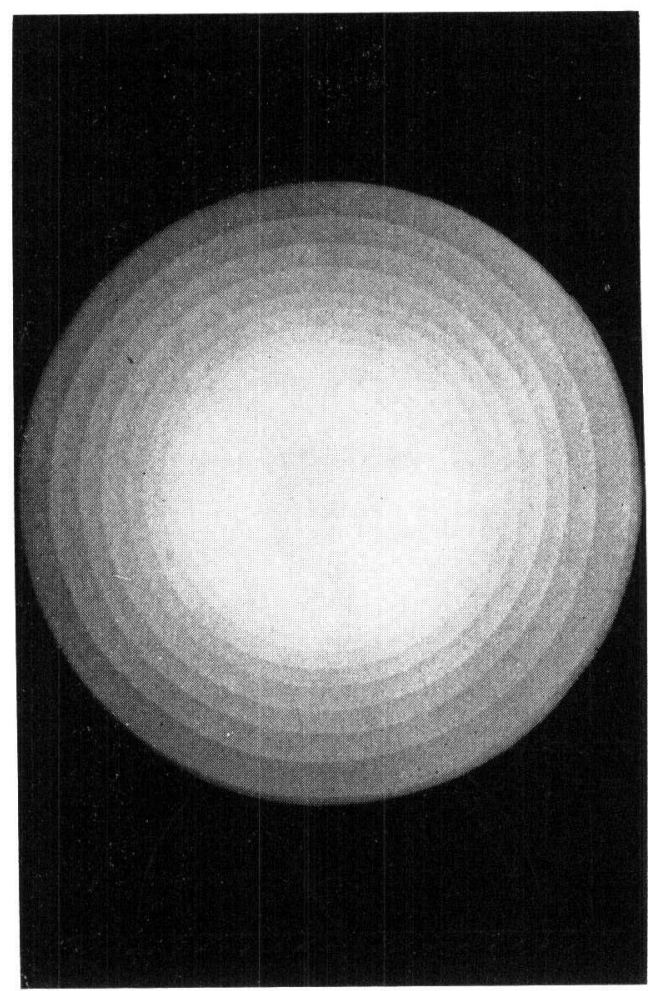

FIG. 5. - La cale optique «rayonnement diffus 》 $(R \mathrm{f})$

FIG. 5. - The optical wedge used to study the «diffuse site factor» (Rf)

Mesure du rayonnement diffus Rf. - Comme pour le couvert angulaire simple, elle s'opère en deux temps, avec le même matériel (source de lumière homogène et lux-mètre) : 
a) entre la source lumineuse et le lux-mètre sont placés la cale circulaire construite et un négatif vierge ; la lecture est $y_{0}$; est $y$.

b) on interpose la cale circulaire et le négatif hémisphérique à analyser; la lecture

Compte tenu des considérations évoquées dans le $\S 3.221$, on peut admettre que la station étudiée reçoit environ, sur l'ensemble d'une année moyenne, et sous forme de rayonnement diffus :

$$
\mathrm{R} f=\frac{y}{y_{0}} .50000 \mathrm{cal} / \mathrm{cm}^{2} / \mathrm{an} .
$$

\subsection{Mesure du rayonnement direct $\mathrm{R} r$.}

Le rayonnement direct est fourni direstement par le soleil. Celui-ci, d'une part ne brille pas en permanence, d'autre part occupe dans le ciel une position variant constamment. Toutefois, on peut admettre que la probabilité pour que le soleil brille est la même pour toutes les positions qu'il peut prendre dans le ciel, comprises entre la trajectoire au solstice d'été et celle au solstice d'hiver.

Soit une trouée circulaire dans le couvert que l'on désire analyser, de surface $\Delta S$ égale à la surface apparente du soleil, située à un angle $t$ par rapport au zénith (fig. 6). L'éclairement $\mathrm{E}$ en $\mathrm{P}$ (lumière d'origine diffuse excluse) est, lorsque le soleil apparaît dans cette direction :

$$
\mathrm{E}=\frac{\mathrm{I} \cos t}{r^{2}}=\mathrm{I} \cos t \quad \text { avec } \quad \mathrm{I}=\mathrm{L} . \Delta \mathrm{S} .
$$

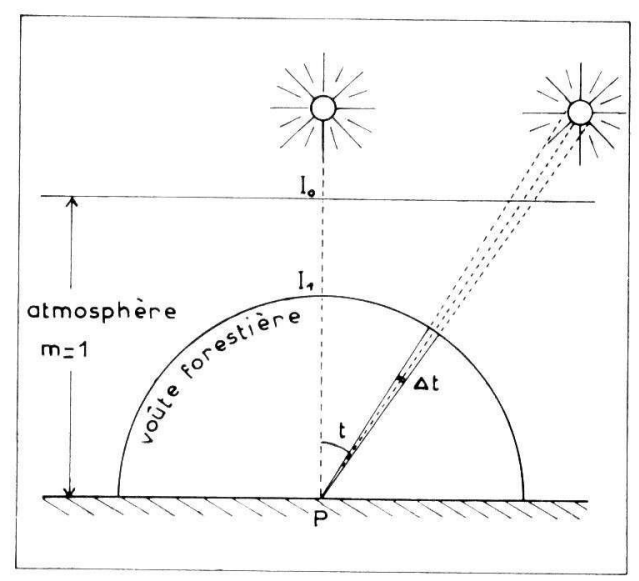

Fig. 6. - Schéma pour l'étude du rayonnement direct $R \mathrm{r}$ (explications dans le texte)

FIG. 6. - Scheme to study the «direct site factor» $R \mathrm{r}$ (explanations in the text)

I est l'intensité de la source lumineuse soleil ; elle n'est pas constante, mais fonction elle-même de $t$. 
On sait que :

$\mathbf{I}=$ Io.$e^{-l \cdot m}$.

Io : intensité solaire hors de l'atmosphère (environ $1,980 \mathrm{cal} / \mathrm{cm}^{2} / \mathrm{mm}$ ).

$m=$ épaisseur atmosphérique traversée par les rayons lumineux (pour 1 atmosphère, $m=1$ ).

$l=$ coefficient d'absorption de l'air, qui dépend de multiples facteurs d'extinction (diffusion par les aérosols, absorption sélective par les constituants fixes, absorption sélective par la vapeur d'eau).

Lorsque le soleil est au zénith $(m=1)$, pour une épaisseur d'eau condensable moyenne $(w=0,5 \mathrm{~cm})$ et un coefficient de trouble de l'atmosphère moyen $(\mathrm{B}=0,05)$, on a (selon Perrin de Brichambaut) :

$$
\begin{aligned}
& I_{1}=1,476 \mathrm{cal} / \mathrm{cm}^{2} / \mathrm{mm} . \\
& \frac{I_{0}}{I_{1}}=\frac{1,980}{1,476}=1,341 .
\end{aligned}
$$

Si l'on prend $\mathrm{I}_{1}$ comme unité, il vient :

$$
1=\text { Io } e^{-l}, \quad \text { d'où : } e^{l}=\text { Io }=1,341 .
$$

Donc : $\mathbf{I}=1,341 \cdot(1,341)^{-m}=1,341^{1-m}$.

Revenons à l'éclairement au point $\mathrm{P}$ :

$$
\mathrm{E}=(1,341)^{1-m} \cdot \cos t \text {. }
$$

Comme pour la mesure du rayonnement diffus, on peut imaginer de superposer au négatif hémisphérique une cale circulaire qui, dans ce cas, ne sera pas exactement sinusoïdale, mais dont le coefficient de transmission $\mathrm{T}$ variera selon la loi :

$$
\mathrm{T}=(1,341)^{1-m} \cdot \cos t \text {. }
$$

Construction de la cale «rayonnement direct». - Il faut d'abord préciser que cette cale sera opacifiée (à l'aide d'un cache adapté) dans les zones correspondant aux régions du ciel non comprises entre les trajectoires du soleil aux solstices d'été et d'hiver.

Ce cache sera construit en fonction de la latitude où doivent être faites les mesures. Des tables donnent, pour une latitude donnée, la position exacte du soleil dans le ciel, pour chaque heure des journées de solstice d'été et d'hiver. Le tableau 1 fournit les éléments pour une latitude de $48^{\circ}$.

Remarquons tout de suite qu'il sera nécessaire, lors de la mesure de $\mathrm{R} r$, d'orienter soigneusement la photographie hémisphérique par rapport au cache.

Comme pour la cale $\mathrm{R} f$, les rayons $t_{n}$ des divers cercles empilés les uns sur les autres pour former le négatif de la cale $\mathrm{R} r$ sont tels que :

$$
\begin{aligned}
& (1,341)^{1-m} \cdot \cos t=1-\frac{\mathrm{To}^{n}+\mathrm{To}^{n-1}}{2} \\
& m=f(t) .
\end{aligned}
$$


TABLEAU 1 - TABLE 1

Azimut et hauteur sur l'horizon du soleil aux solstices d'été et d'hiver, à la latitude de $48^{\circ}$

Azimuth and sun height above the horizon at summer and winter solstices, in latitude $48^{\circ}$

\begin{tabular}{|c|c|c|c|c|c|c|c|c|c|}
\hline 21 juin & $\begin{array}{l}4 \mathrm{~h} 05- \\
19 \mathrm{~h} 55\end{array}$ & $5 \mathrm{~h}-19 \mathrm{~h}$ & $6 h-18 h$ & $7 \mathrm{~h}-17 \mathrm{~h}$ & $8 h-16 h$ & $9 h-15 h$ & $10 \mathrm{~h}-14 \mathrm{~h}$ & $11 h-13 h$ & $12 \mathrm{~h}$ \\
\hline Azimut $\ldots \ldots \ldots$ & $\pm 126,5^{\circ}$ & $\pm 116,5^{\circ}$ & $\pm 106,2^{\circ}$ & $\pm 95,5^{\circ}$ & $\pm 84,9^{\circ}$ & $\pm 71,6^{\circ}$ & $\pm 54,9^{\circ}$ & $\pm 31,3^{\circ}$ & $0^{\circ}$ \\
\hline $\begin{array}{l}\text { Hauteur sur l'ho- } \\
\text { rizon } \ldots \ldots \cdots \cdots \cdots\end{array}$ & $0^{\circ}$ & $7,9^{\circ}$ & $17,2^{\circ}$ & $27,1^{\circ}$ & $37,1^{\circ}$ & $46,9^{\circ}$ & $55,9^{\circ}$ & $62,7^{\circ}$ & $65,4^{\circ}$ \\
\hline
\end{tabular}

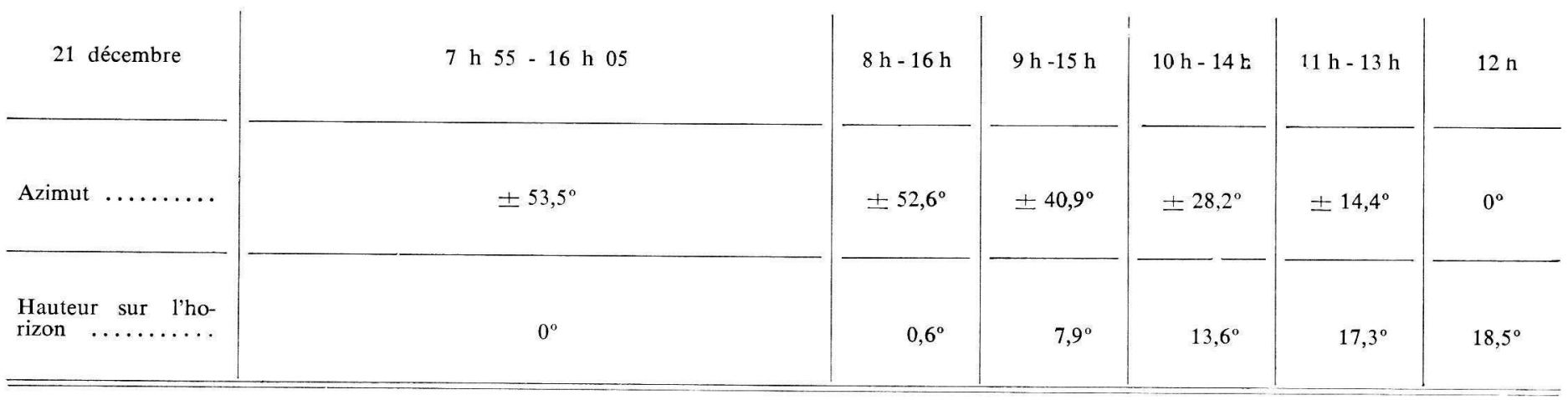


Perrin de Brichambaut donne, pour une pression de $1.000 \mathrm{mb}$, et une tempéra. ture de $10^{\circ} \mathrm{C}$, la masse atmosphérique $m$ en fonction de la hauteur du soleil, compte tenu de la courbure terrestre et de la courbure des rayons lumineux (due aux variations de l'indice de réfraction de l'air avec l'altitude). Il est donc possible de construire la courbe $(1,341)^{1-m} \cdot \cos t$ (fig. 7), qui permet ensuite de déterminer les valeurs $t_{n}$.

Le négatif obtenu est photographié de façon à obtenir la cale définitive de diamètre 23,2 $\mathrm{mm}$. Le même problème que précédemment se pose pour le choix de la meilleure exposition. On opère de façon semblable :

- Qo $=K \frac{\pi^{3}}{4}$

$-\mathrm{Q}=2 \pi \mathrm{K} \int_{0}^{\frac{\pi}{2}} t \cdot(1,341)^{1-m} \cdot \cos t \cdot d t$.

A partir de la courbe de la fig. 7, nous avons construit la courbe t. $(1,341)^{1-m} \cdot \cos t$, et, par mesure, on obtient :

$\mathrm{Q}=2 \pi \mathrm{K} \cdot 0,452$,

donc: $\frac{\mathrm{Q}}{\mathrm{Qo}}=\frac{8 \pi \cdot 0,452}{\pi^{3}}=0,366$.

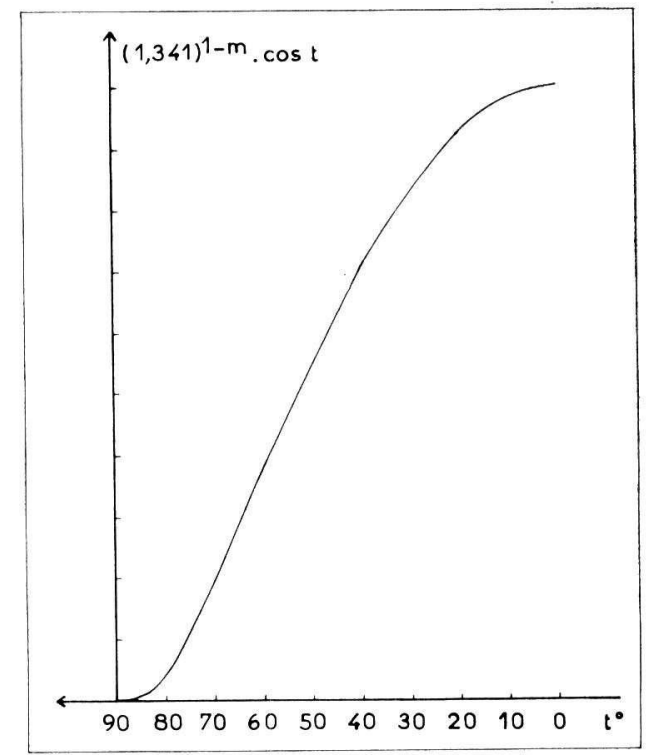

FIG. 7. - La courbe $(1,341)^{1-\mathrm{m}} \cdot \cos \mathrm{t} ; \mathrm{m}=\mathrm{f}(\mathrm{t})$

Fig. 7. - The curve $(1,341)^{1-\mathrm{m}}$. cos $\mathrm{t} ; \mathrm{m}=\mathrm{f}(\mathrm{t})=$ atmosphere mass that the sun rays pass through

La photographie de la fig. 8 représente la cale $\mathrm{R} r$ définitive, munie de son cache, valable pour les régions proches de $48^{\circ}$ de latitude. 
Mesure du rayonnement direct $\mathrm{R} r$. - La mesure s'opère là encore en deux temps :

a) avec la cale $\mathrm{R} r$, munie de son cache, et un négatif vierge ; la lecture est $y_{0}^{\prime}$,

b) avec la cale $\mathrm{R} r$, munie de son cache, et le négatif hémisphérique à analyser, soigneusement orienté par rapport au cache ; la lecture est $y^{\prime}$.

On peut admettre que la station étudiée reçoit environ, sur l'ensemble d'une année moyenne, et sous forme de rayonnement direct:

$$
\mathrm{R}_{r}=\frac{v^{\prime}}{y_{o}^{\prime}} \cdot 50.000 \mathrm{cal} / \mathrm{cm}^{2} / \mathrm{an} .
$$

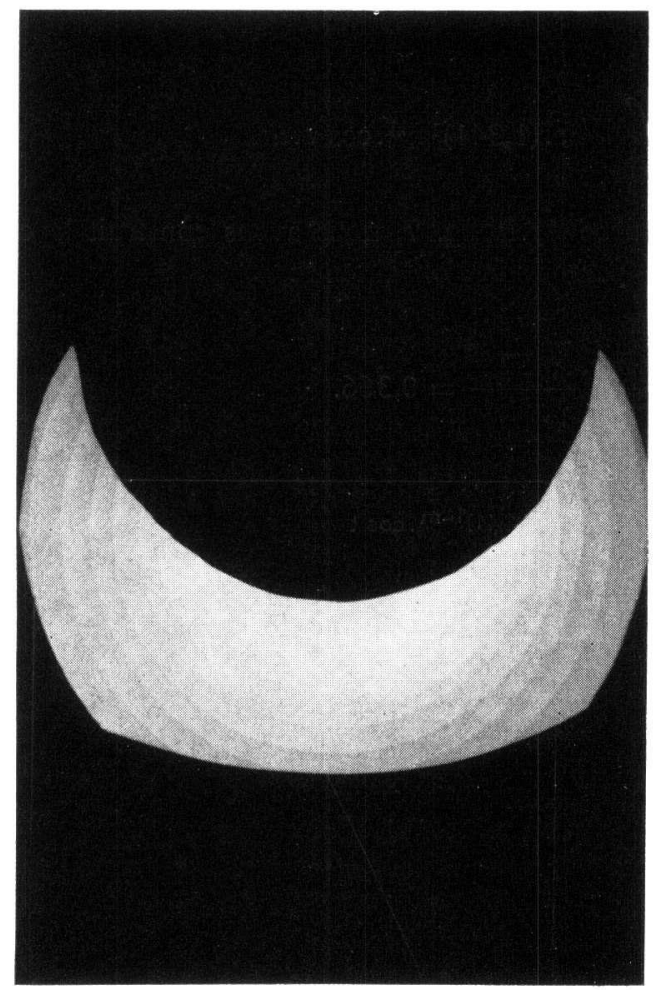

Fig. 8. - La cale optique «rayonnement direct» $\left(R_{n}\right)$

FIG. 8. - The optical wedge used to study the «direct site factor $\gg\left(R_{n}\right)$

\subsection{Détermination du rayonnement global au sol.}

Le rayonnement global est donc:

$\mathbf{R}=\mathbf{R}_{f}+\mathbf{R}_{r}=\left(\frac{y}{y_{0}}+\frac{y^{\prime}}{y_{0}^{\prime}}\right) .50 .000 \mathrm{cal} / \mathrm{cm}^{2} / \mathrm{an}$.

A titre d'exemple, voici les résultats des mesures faites sur la photographie de la figure 2 (taillis vieilli de Hêtre, très dense) :

$\mathbf{R}_{r}=2.200 \mathrm{cal} / \mathrm{cm}^{2} / \mathrm{an}$, soit $4,4 \%$ (par rapport au plein découvert).

$\mathbf{R}_{f}=2.800 \mathrm{cal} / \mathrm{cm}^{2} / \mathrm{an}$, soit $5,6 \%$.

Total $\mathrm{R}=5.000 \mathrm{cal} / \mathrm{cm}^{2} /$ an $(5 \%)$. 


\section{IV. - RÉFLEXION SUR LA VALEUR DES MESURES}

\section{1. - Couverts de morphologie stable (à l'échelle d'une année)}

Il s'agit des couverts à base de résineux ou de feuillus à feuilles persistantes.

Un des principes de base de la technique proposée est de rechercher dans la photographie un effet de contre-jour aussi complet que possible. Ceci permet de comparer des clichés pris dans des conditions très différentes d'éclairement extérieur au peuplement. En contrepartie, cela revient à négliger le rayonnement parvenant au sol à travers la masse végétale et, à la limite, à accorder le même pouvoir d'arrêt, vis-àvis de la lumière, à une seule épaisseur de feuilles qu'à un couvert très fourni.

Ces mesures, prises telles quelles, sont donc automatiquement approchées par défaut. Dans l'état actuel de nos travaux, les chiffres obtenus devront donc être avant tout considérés comme des indices, permettant de comparer des stations entre elles, plus que des mesures valables dans l'absolu. C'est d'ailleurs le but que nous nous étions fixés à l'origine de cette étude.

Il reste cependant possible d'étalonner la technique décrite, afin de corriger les valeurs obtenues. Des mesures stationnelles de longue durée sont actuellement entreprises au C.N.R.F., avec l'aide de lux-mètres totalisateurs et de thermopiles munies d'intégrateurs. Placés dans divers milieux forestiers, ces appareils, après avoir fonctionné pendant une période suffisamment longue (plusieurs années si possible) fourniront des chiffres qui seront comparés avec ceux obtenus instantanément par les photographies hémisphériques.

\section{2. - Couverts de morphologie variable (à l'échelle d'une année)}

Il s'agit des couverts à bases de feuillus à feuilles caduques.

Sauf étude particulière (flore vernale, par exemple), les photographies sont prises lorsque la foliaison est totale. Mais on introduit de ce fait une nouvelle cause d'erreur en négligeant l'apport supplémentaire d'énergie en phase défeuillée, où le couvert angulaire est bien plus faible. Une correction est donc nécessaire. De plus, il semble à peu près établi que les couverts feuillus, en été, et surtout par temps ensoleillé, réémettent vers les sous-bois une lumière faiblement enrichie en rayonnement infrarouge (à partir de $750 \mathrm{~nm}$ ). Il peut en résulter de légères discordances entre l'éclairement lumineux relatif et l'éclairement énergétique relatif.

Là encore, les mesures de longue durée, évoquées dans le paragraphe 4.1, devraient fournir les éléments, soit pour une correction unique, soit pour parvenir au bilan global par l'intermédiaire de deux photographies prises, l'une en phase feuillée, l'autre en phase défeuillée (à condition de connaître l'énergie reçue du soleil hors peuplement pendant chacune de ces deux phases).

Il nous paraît nécessaire de déterminer expérimentalement les corrections, selon le processus décrit ci-dessus, pour cinq types schématiques de peuplements : feuillus à feuilles caduques du type Chêne (essence de lumière), feuillus à feuilles caduques du type Hêtre (essence d'ombre), feuillus à feuilles persistantes, résineux du type Sapin, Epicéa, Douglas... (Abiétées), résineux du type Pin. 


\section{3. - Conclusion}

En attendant les résultats de ces mesures stationnelles de longue haleine, les photographies hémisphériques apporteront, en toute fazilité, d'utiles données chiffrées, et objectives, permettant de comparer entre el!es de nombreuses stations. Précisons encore une fois que ces comparaisons ne seront pleinement satisfaisantes que si les strates arborescentes sont à base de la même essence ou du même type d'essence (du point de vue de la morphologie).

Précisons, d'autre part, que nous nous sommes implicitement référés, dans notre texte, à l'unité de surface horizontale pour l'étude des stations. Il est bien évident que la pente et l'exposition apportent des éléments de correction parfois très importants. Les évaluer avec précision aboutit à des formulations extrêmement complexes et pratiquement inutilisables.

Nous nous attachons actuellement à mettre au point une technique de correction simple, mais suffisamment précise pour les besoins de la phyto-écologie. En attendant, il est indispensable de compléter la mesure du rayonnement global telle que nous l'avons exposée, par celles de la pente et de l'exposition.

Reçu pour publication en mai 1971.

\section{REMERCIEMENTS}

Je tiens à exprimer ici mes plus vifs remerciements à M. le Conservateur Roussel, qui m'a aidé de ses encouragements lorsqu'est née l'idée de cette technique et qui n'a cessé depuis de me prodiguer l'aide précieuse de ses conseils.

\section{SUMMARY}

\section{A NEW METHOD USING HEMISPHERICAL PHOTOGRAPHS TO} ASSESS LIGHT CLIMATE IN FOREST

Light climate is, among the ecological data, one of the most important, but also the most difficult to measure in forest.

The method we propose, uses hemispherical photographs (made with a canopy camera, wide angle $180^{\circ}$, focal length $7,5 \mathrm{~mm}$ ). During the photography, the camera is set horizontally. The film which is used gives high contrasts (Kodak Microfile), in order to have only blacks (gaps in the canopy) and whites (foliage).

The negatives which are obtained must be inverted; one measures the transmission factor of the whole transparent photograph (fig. 2) to obtain the «angular shelter » of the studied canopy.

To have an approached value of the annual mean energetical radiation, one must distinguish the «diffuse site factor» $(R f)$ and the «direct site factor» $(\mathbf{R} r)$ (ANDERSON, 1964). To this end, two different measures are made, by setting on the transparent photograph a « diffuse optical wedge » (fig. 5), then a «direct optical wedge» (fig. 8), which are both as wide as the transparent photograph.

The « $R f$ optical wedge 》 is round and sinusoidal; it corrects the transmission factor of the transparent photograph to take into account the height of the gaps in the canopy above the horizon. From its centre to its periphery, the transmission factor of the $\mathbf{R} f$ optical wedge varies according to the law: $\mathrm{T}=\operatorname{cost}(t=$ distance from the centre of the photograph, in "). 
The « Rr optical wedge » has to take into account the portion of the sky in which the sun occurs during one year, in the studied country (fig. 8). In the corresponding zone, its transmission factor varies according to a law which is a little more complicated; this one takes into account, not only the height of the gaps above the horizon, but also the atmospheric mass $m$ :

$$
\mathrm{T}=(1,341)^{1-m} \cdot{ }^{m} \cdot \cos . t
$$

We have now to study the accuracy of this method, by comparison with measures made with light integrators during long periods. We have also to imagine a method to correct the measures according to the slope and the exposure of the studied areas.

\section{ZUSAMMENFASSUNG}

EINE NEUE VERWENDUNGSTECHNIK VON WEITWINKELPHOTOGRAFIEN

ZUR MESSUNG DES LICHTKLIMAS IM WALDE

Das Lichtklima ist einer der wichtigsten ökologischen Faktoren, es ist im Walde jedoch äusserst schwer zu erfassen.

In der vom Autor vorgeschlagenen Methode werden Weitwinkelaufnahmen (SuperWeitwinkelobjektiv mit $180^{\circ}$ Offnung) verwendet. Die Kleinbildkamera wird horizontal, gegen den Zenith gerichtet, aufgestellt. Es wird ein Film mit sehr grossem Kontrast verwendet, um nur schwarze (Offnungen im Kronendach) oder weisse (Vegetation oder Relief) Abbildungen $\mathrm{zu}$ erzielen.

Von den so erhaltenen Negativen wird ein Kontaktposotiv angefertigt und der globale Transmissionskoeffizient des Diapositivs gemessen (Abb. 2), um den «Beleuchtungsgrad 》 (couvert angulaire) des Standorts zu bestimmen.

Um einen Näherungswert der mittleren jährlichen Globalstrahlung zu erhalten, muss zwischen Himmelsstrahlung $(\mathbf{R} f)$ und Sonnenstrahlung $(\mathbf{R} r)$ unterschieden werden. Es müssen zwei verschiedene Messungen durchgeführt werden, wobei jeweils ein optischer Keil für die Himmelsstrahlung (Abb. 5) und für die Sonnenstrahlung (Abb. 8) veiwendet werden; beide Keile entsprechen dem Format des Diapositivs und werden ihm jeweils vorgeschaltet.

Der Rf-Keil korrigiert den Transmissionskoeffizienten des Diapositivs, um der Höhe von Kronendachlücken über dem Horizont Rechnung zu tragen. Der Keil ist kreisförmig und sein Transmissionskoeffizient ändert sich vom Zentrum zum Rand nach dem Gesetz $\mathrm{T}=\operatorname{cost}(t=$ Zenithabstand in Graden $)$.

Der R $r$-Keil trägt den Sonnenbahnen innerhalb einer bestimmten Region Rechnung (siehe Abb. 8). In dem entsprechenden Bereich ändert sich der Transmissionskoeffizient des Keiles nach dem Gesetz $T=(1,341)^{1-m} \cdot \cos . t$, das sowohl die Horizonthöhen der Kronendachlücken, als auch die Luftmasse und ihren Absorbtionskoeffizienten berücksichtigt.

Es bleibt weiteren Untersuchungen vorbehalten die Genauigkeit dieser Methode durch Vergleiche mit langen Messperioden der Globalstrahlung zu überprüfen und geeignete einfache Methoden zur Berïcksichtigung der Hangneigung und Exposition auszuarbeiten.

\section{RÉFÉRENCES BIBLIOGRAPHIQUES}

Anderson M. C., 1964. Light relations of terrestrial plant communities and their measurement. Biol. Rev., 39, 425-486.

Anderson M. C., 1964. Studies of the woodland light climate. I. Photographic computation of light conditions. J. Ecol., 52, 27-41.

Aussenac G., BeCKer M., 1968. Ecologie d'un massif sur sols hydromorphes : la forêt de Charmes (Vosges). Contribution à la mise au point d'une méthode d'étude dynamique du milieu forestier. Ann. Sci. forest., 25, 4, 291-332.

Becker M., 1969. Le Hêtre (Fagus silvatica L.) et ses problèmes en forêt de Villers-Côtterets (Äisne). Contribution à la mise au point d'une méthode dynamique d'étude écologique du milieu forestier. Ann. Sci. forest., 26, 2, 141-182. 
BonHomme R., 1970. Application de la technique des photographies htmisphériques in situ à la mesure de l'indice foliaire. In Techniques d'étude des facteurs physiques de la biosphère, 501-6, I.N.R.A., Paris.

Bonfromme R., 1970. Surface relative des taches de soleil dans la végétation. In Techniques d'étude des facteurs physiques de la biosphère. 99-104, I.N.R.A., Paris.

Brown H. E., Wordley D.P., 1965. Some applications of the canopy camera in forestry. J. For., 63, 674-680.

Evans G. C., Coоmbe D. E., 1959. Hemispheral and woodland canopy photography and the light climate. J. Ecol., 47, 103-113.

DuCREY M., 1970. Utilisation des photographies hémisphériques pour l'évaluation de la densité du couvert forestier et pour le calcul de la perméabilité au rayonnement solaire. Note provisoire, C.N.R.F., Nancy.

Madgwick H. A. I., Brumfield G. L., 1969. The use of hemispherical photographs to assess light climate in the forest. J. Ecol., 57, 2, 537-542.

Perrin de Brichambaut Ch., 1963. Rayonnement solaire et échanges radiatifs naturels. Monographies de météorologie. Gauthier-Villars, Paris.

PiCard J. F., 1970. Les forêts sur Rhétien dans le département des Vosges. Nouvelle contribution à la mise au point d'une méhode dynamique d'étude phyto-écologique du milieu forestier. Thèse, $3^{\mathbf{e}}$ cycle, Nancy.

Roussel L., 1952. Recherches théoriques et pratiques sur la répartition en quantité et en qualité de la lumière dans le milieu forestier. Influence sur la végétation. Ann. Ec. nation. Eaux et Forêts et St. Rech. Expér., 13, 1, 295-400.

Roussel L., 1962. Etude théorique élémentaire de la trouée et de la bande. Bull. Soc. forest. Franche-Comté, 2, 552-560.

Roussel L., 1968. Les radiations naturelles et la forêt. Bull. Soc. forest. Franche-Comté, 79 p. 